\title{
SUR LE CUT-LOCUS D'UNE VARIÉTÉ PLONGÉE
}

\author{
R. THOM
}

\section{Préliminaire}

Soit $C^{r}(M)$ l'espace des fonctions réelles de classe $\mathscr{C}^{r}$ sur une variété différentiable compacte $M$ (muni de la topologie $\mathscr{C}^{r}, r>0$ ).

On sait que cet espace fonctionnel est muni d'une stratification canonique, définie par exemple par les orbites du groupe Diff $\boldsymbol{M} \times$ Diff $\boldsymbol{R}$. Rappelons que jusqu'en codimension six, les orbites définissent une telle stratification; en codimension plus élevée apparaissent des modules, qui obligent à concentrer les orbites en strates (Voir J. N. Mather [2], F. Sergeraert [5]). Nous n'aurons ici à considérer explicitement que des strates de codimension inférieure à deux. On trouve dans J. Cerf [1] une description explicite de ces strates que nous rappelons ici brièvement: $X^{0}$, de codimension zéro, est l'ensemble des fonctions de Morse excellentes (à valeurs critiques toutes distinctes); $X^{1}$, de codimension un, est formée des fonctions dont deux seulement des valeurs critiques sont égales, ou qui admettent un seul point critique de type $f=x^{3}+$ forme quadratique non dégénérée. $X^{2}$, strate de codimension deux, formée des fonctions dont trois valeurs critiques seulement sont égales, ou des fonctions dont un point critique d'index un a même valeur qu'un point critique ordinaire, ou des fonctions dont un point critique est de type $x^{4}+\sum x_{i}^{2}$.

On observera que la partie lisse de l'ensemble $X^{1}$ (dit ensemble de bifurcation de $C^{r}(M)$ ) se décompose en certains ensembles $X_{i, j}$, ensemble des fonctions $f$ qui admettent un point critique d'indice $i$ et un point critique d'indice $j$ d'égale valeur, les autres points critiques étant génériques. On appellera $X_{0,0}^{m} l^{\prime}$ ensemble des fonctions qui admettent leur minimum absolu en deux points critiques simples distincts.

Définition. Le sous-ensemble fermé $W \subset C^{r}(M)$ tel que toute fonction $g \mathrm{du}$ complémentaire $C^{r}-W$ atteint son minimum absolu en un point critique ordinaire sera dit l'ensemble de Maxwell de l'espace fonctionnel. Ce complémentaire est évidemment partout dense dans $C^{r}(M)$.

Proposition 1. L'ensemble $W$ est l'adhérence de l'ensemble $X_{0,0}^{m}$.

Une fonction $g \in W$ atteint son minimum absolu en deux points $c_{1}, c_{2}$ distincts au moins, ou en un minimum dégénéré unique. Dans le premier cas, on approchera $g$ par une fonction $f$ qui atteint son minimum absolu en deux points simples $x_{1}, x_{2}$ voisins de $c_{1}, c_{2}$ respectivement, donc dans $X_{0,0}^{m}$. Dans le second cas, le minimum, dans une carte locale, s'écrit $g=x_{1}^{2 s}+h\left(x_{2}, \cdots, x_{n}\right)$, entier $s>0$ et $\operatorname{dg} h \geq 2$. 
En ajoutant à $g$ une fonction de la forme $x_{1}^{4}+\sum x_{j}^{2}$, on approche arbitrairement près $g$ par une fonction dont le minimum absolu est atteint en un point de type $x_{1}^{4}+\sum x_{j}^{2}, j \geq 2$, c'est à dire une fonction de $X^{2}$; mais ces fonctions sont dans l'abhérence de $X_{0,0}^{m}$ comme le montre la fonction $x^{4}-a x^{2}, a>0$ arbitrairement petit.

Il résulte de la théorie générale des singularités de fonctions différentiables (théorie du "déploiement universel") que l'étoile d'une strate de la stratification canonique (de codimension finie) admet un modèle local défini par des ensembles semi-algébriques, donc localement triangulable; cf. [2]. Il en va de même de l'ensemble $W$, sous-ensemble stratifié de cette stratification.

De plus, comme pour toute stratification définie par les orbites d'une action de groupe, on a la propriété de rigidité locale (dans le bon cas de codimension $\leq 6)$ : Tout champ de vecteur $X$ d'une strate $U$ s'étend localement en un champ de vecteur $\hat{X}$ tangent aux strates de la stratification situées dans l'étoile de $U$. Il en résulte qu'on peut définir la notion d'une application différentiable $F: \boldsymbol{R}^{k} \rightarrow C^{r}(M)$ transverse sur l'ensemble de Maxwell $W$. (On suppose ici $k \leq 6$ pour rester génériquement dans le "bon cas": cette hypothèse n'est sans doute pas nécessaire ...). Si $F$ est transverse sur $W$, la contre-image $F^{-1}(W)$ est un ensemble stratifié, doté de la même propriété de rigidité des étoiles. . . Ces étoiles ont en particulier des modèles locaux semi-algébriques, donc polyèdraux.

\section{Cut-locus d'une variété plongée dans l'espace euclidien}

Soit $f$ une application différentiable d'une variété $M^{n}$ dans l'espace euclidien $\boldsymbol{R}^{n+p}$. On associe à cette application $f$ une application $\Phi(f)$ de l'espace ambiant $\boldsymbol{R}^{n+p}$ dans $C^{r}(M)$, dite: champ des fonctions distances associé à $f$, par la formule: $[\Phi(f)(x)](y)=d^{2}(f(x), y), d$ distance euclidienne. On voit que $\Phi(f)$ prend ses valeurs dans l'ensemble $C^{+}$des fonctions positives sur $M$.

Définition. On appelle cut-locus $K(f)$ de l'application $f: M \rightarrow \boldsymbol{R}^{n+p}$ l'ensemble contre-image par $\Phi(f)$ de l'ensemble de Maxwell $W \subset C^{r}(M)$.

Enonçons alors la conjecture:

Conjecture (CL). Pour presque toute application f de $M$ dans $\boldsymbol{R}^{n+p}$ (au sens de la $\mathscr{C}^{k}$-topologie), l'application $\Phi(f)$ de $\boldsymbol{R}^{n+p}$ dans $C^{k}(M)$ est transverse sur l'ensemble de Maxwell $W$.

J. N. Mather a annoncé un résultat semblable concernant le cut-locus d'un point dans une variété riemannienne, lorsqu'on fait varier la métrique. Très vraisemblablement, sa technique de démonstration pourra s'appliquer à la conjecture ci-dessus. Pour le cas $n=1,2, p=1$, la conjecture peut être démontrée par réduction au lemme de transversalité, appliqué dans l'espace des jets et des multijets (au sens de Mather) de l'application $f$.

La conjecture $(\mathrm{CL})$ permet de définir de manière précise les singularités génériques d'un cut-locus.

L'application $f$ a un cut-locus générique, si le champ $\Phi(f)$ associé est trans- 
verse sur l'ensemble de Maxwell $W$. En ce cas, la cut-locus $K(f)=\Phi(f)^{-1}(W)$ a lui-même une structure stratifiée, et les modèles locaux, semi-algébriques, des incidences des strates sont les sections transversales des modèles locaux définis sur $W$.

L'ensemble de Maxwell $W$ comporte trois sortes de strates (cf. [6]):

i) des strates de bifurcation. Ensembles des fonctions dont le minimum absolu est atteint en un minimum dégénéré d'un certain type (algébriquement isolé).

ii) des strates de conflit. Ensembles des fonctions qui atteignent leur minimum absolu en $k$ minima simples $c_{1}, c_{2}, \cdots, c_{k}$.

iii) Strates mixtes. Même définition qu'en ii), lorsqu'au moins un des minima $c_{j}$ est dégénéré.

Les incidences des strates de conflit entre elles sont aisées à décrire: dans l'espace $\boldsymbol{R}^{k}$ de coordonnées barycentriques $t_{0}, t_{1}, \cdots, t_{k}$, il s'agit toujours de variétés linéaires définies par les équations et inéquations: $t_{1}=t_{2}=\cdots=$ $t_{s}<\operatorname{Inf}\left(t_{s+1}, \cdots, t_{k}\right)$. En général, une strate de bifurcation est dans l'adhérence de strates de conflit (Proposition 1). Le modèle local de l'étoile est décrit par un ensemble de Maxwell défini localement dans le déploiement universel du minimum dégénéré. Par exemple, la singularité $f=x^{4}$ admet pour déploiement universel $F=x^{4} / 4+u x^{2} / 2+v x$. Dans le plan $O u v$ du déploiement, la strate $X^{2}$ de bifurcation est l'origine $u=v=0$. La strate de conflit incidente est l'ensemble de Maxwell $v=0, u<0$. (Cf. la théorie de la catastrophe de RiemannHugoniot [6].)

Cas des petites dimensions. A) $n=1, p=1$ (Courbes planes). Il y a deux strates de conflit: l'arc simple, de dimension un, défini par l'égalité de deux minima simples, et le point triple, obtenu lorsque trois minima simples sont égaux.

Il y a une seule strate de bifurcation: le point-fronce $X^{2}$ defini par un minimum de type $x^{4}$. Ce point est l'extrémité libre d'un arc de conflit. Il n'y a pas de strates mixtes, puisque la plus simple, obtenue en écrivant qu'un minimum simple et un minimum de type $x^{4}$, est de codimension trois, donc non générique.

Il en résulte que génériquement le cut-locus d'une courbe plane est un graphe, dont les seuls sommets sont des points triples, ou des sommets libres. Ce résultat est bien connu dans le cas du cut-locus d'une surface.

B) $n=2, p=1$. Il y a trois strates de conflits : la nappe, de dimension deux où deux minima simples sont égaux; l'arête triple (suspension du point triple), où trois minima simples sont égaux; et le point quadruple; où quatre minima sont égaux. L'étoile d'un point quadruple s'obtient en joignant le barycentre d'un 3-simplexe (tétraèdre) aux sommets et arêtes du bord.

Il n'y a qu'une seule strate de difurcation: l'arête $X^{2}$, de dimension un, codimension deux, donnée par les fonctions dont le minimum absolu est de type $x^{4}$. La singularité de type $x^{5}$ (Oueue d'aronde) n'apparait pas, car ce n'est pas un minimum. L'arete $X^{2}$ est le bord libre d'une nappe de conflit. 
Il existe une seule strate mixte, de codimension trois, dimension zéro: ensemble des fonctions qui atteignent leur minimum en un minimum simple et un minimum de type $X^{2}$. Un tel point est l'extrémité libre d'une arête triple, et la nappe de conflit s'exfolie en donnant naissance à une nouvelle nappe de bord $X^{2}$. Ce point est également le point d'arrêt de l'arête $X^{2}$ (cf. Fig. 1).

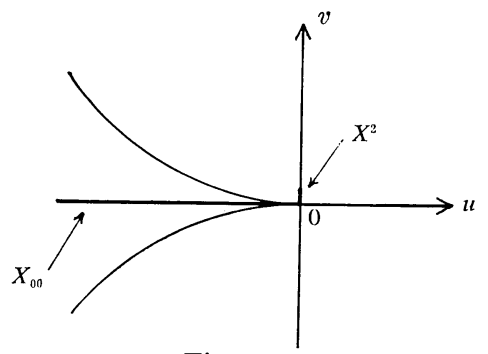

Fig. 1.

Par suite, presque toute surface dans $\boldsymbol{R}^{3}$ admet pour cut-locus un polyèdre de dimension deux, qui ne comporte que des arêtes triples ou des arêtes libres, et dont les sommets ont la configuration décrite ci-dessus.

\section{Propriétés génériques du cut-locus}

Disons qu'une application $F$ d'une variété $X$ dans $C(M)$ est de classe $\mathscr{C}^{r}$, si l'application $G: X \times M \rightarrow R$ définie par $G(x, m)=F(x) \cdot(m)$ est elle-même de classe $\mathscr{C}^{r}$. Il est immédiat de voir que, dans ces conditions, l'application $\Phi(f)$ : $\boldsymbol{R}^{n+p} \rightarrow C^{r}(M)$ est différentiable, et dépend differentiablement de $f$. Par suite, si une application $f: M \rightarrow \boldsymbol{R}^{n+p}$ est telle que $\Phi(f)$ est transverse sur $W$, il en ira de même pour toute application $\left(f^{\prime}\right)$ assez voisine de $f$ dans la $\mathscr{C}^{r}$-topologie. En raison du théorème d'isotopie des sections transversales d'ensembles stratifiés, les cut-loci $K(f), K\left(f^{\prime}\right)$ sont isotopes dans $\boldsymbol{R}^{n+p}$. Si on munit $M$ d'une structure algbérique réelle (par le théorème de Nash), alors $f$ peut être approchée par une application $f^{\prime}$ qui est un morphisme de Nash (algébrique). Alors l'ensemble $K\left(f^{\prime}\right)=\Phi\left(f^{\prime}\right)^{-1}(W)$ est un ensemble semi-algébrique de $M$. Il existe par suite une subdivision simpliciale de $M$ contenant $K\left(f^{\prime}\right)$ comme sous-complexe. De là résulte que, génériquement, le cut-locus $K(f)$ d'une application $f$ est rétracte par déformation d'un de ses voisinages.

Lemme 1. Soit $Q=\sum x_{i}^{2}$ le carré de la distance euclidienne dans $\boldsymbol{R}^{k}$; une application différentiable $g: \boldsymbol{R}^{s} \rightarrow \boldsymbol{R}^{k}, s \leq k$, telle que $g(0)=0$, est régulière en 0 si et seulement si la fonction induite $Q^{\prime}=Q \circ g$ est une forme quadratique non dégénérée.

Il est immédiat que si $g$ est régulière en 0 (de rang $s$ ), $Q^{\prime} \circ g$ est quadratique non dégénérée. Si $g$ est singulière, il existe un vecteur $Z \neq 0$ tangent à 0 dans le noyau de $j^{1}(g)$; alors $Q^{\prime}(Z, Z)=Q \circ g(Z, Z)=Q(\dot{g}(Z), \dot{g}(Z))=0$.

Définition. Si $f$ est une application différentiable de $\boldsymbol{M}^{n}$ dans $\boldsymbol{R}^{n+p}$, on 
appelle partie lisse, notée $S m(f)$, de l'image $f(M)$, l'ensemble image des points réguliers de $M$, diminué de la variété de self-intersection de $f$. Autrement dit, si $y \in S m(f), f^{-1}(y)$ ne comporte qu'un point $x$, où $f$ est de rang maximum $n$.

Le complémentaire $f(M)-S m(f)$ sera dit partie singulière de l'image $f(M)$. C'est un sous-ensemble fermé de $f(M)$ (éventuellement identique à $f(M)$ ).

Proposition 2. L'intersection du cut-locus $K(f)$ avec l'image $f(M)$ est la partie singulière de $f(M)$.

En effet, en un point $y$ de la partie lisse $\operatorname{Sm}(f)$ de $f$, la fonction $\Phi(f)(y)=$ $d^{2}(f(x), y)$ atteint son minimum absolu, zéro, au seul point $x_{0}$ tel que $f\left(x_{0}\right)=y$, et, d'après le lemme 1 , ce minimum est simple (non dégénéré). Au contraire, si $z$ appartient à la partie singulière de $f(M)$, la fonction $d^{2}(f(x), z)$ atteint son minimum, zéro, soit en au moins deux points (si $z$ est un point de self-intersection), soit en un minimum dégénéré, si $z$ est image d'un point singulière de $f$ (Lemme 1).

Théoreme 1. Soit $K(f)$ le cut-locus de l'application $f: M \rightarrow \boldsymbol{R}^{n+p}$; alors le complémentaire $\boldsymbol{R}^{n+p}-K(f)$ est "fibré" en p-boules ouvertes sur la partie lisse $\operatorname{Sm}(f)$ de l'application $f$.

En effet, soit $z$ un point du complémentaire $\boldsymbol{R}^{n+p}-K(f)$.

La fonction $d(f(x) ; z)$ prend son minimum absolu en un minimum simple $x^{\prime}=q(z)$. D'après le théorème des fonctions implicites ( $x^{\prime}$ est un minimum simple), $x^{\prime}$ est une fonction différentiable du point $z$, et l'application $q: z \rightarrow$ $x^{\prime}$ est localement de rang $n$. En effect, si la normale $z x^{\prime}$ est minimale pour $z$, elle est aussi minimale pour tout point $y$ situé entre $z$ et $x^{\prime}$ (d'après l'inégalité du triangle); par suite, si l'on considère l'application $\operatorname{Exp}: \boldsymbol{T} \rightarrow \boldsymbol{R}^{n+p}$ du fibré normal $T$ à $S m(f)$ dans $\boldsymbol{R}^{n+p}$, définie par les droites normales à $S m(f)$, on voit que si $u$ est une valeur critique de Exp, deux cas sont possibles: ou la normale minimale issue de $u$ est unique et simple; en ce cas, $u$ admet une contre-image $v$ pour lequel Exp est de rang maximum; par suite l'application $q$ peut être relevée dans $T$ sur un voisinage de $u$ en une application $q$ de rang maximum (projection du fibré normal $T \rightarrow S m(f))$. En ce cas $v$ est extérieur au cut-locus $K(f)$. Si la normale minimale est non unique, ou dégénérée, $v$ appartiént au cut-locus. Ainsi $q$ définit une application surjective de rang maximum de $R^{n+p}-K(f)$ sur $\operatorname{Sm}(f)$. Lorsque $K(f)$ n'a que des singularités génériques, on peut l'encapsuler dans une famille d'hypersurfaces qui le tapissent: il existe un voisinage $J \subset K(f)-f(M)$, et une fonction $H: J \rightarrow R$, telle que $H^{-1}(0)=K(f), d H \neq 0$ sur $J-K(f)$, et telle que $q$ soit de rang maximum sur chacune des hypersurfaces $H^{-1}(\varepsilon), \varepsilon>0$ assez petit. Ceci fait de $q$ une submersion contrôlée et par suite $q$ est une fibration.

Corollaire 1. Le cut-locus $K(f)$ est rétracte par déformation du complémentaire de la partie lisse $\operatorname{Sm}(f)$.

En effet, le fibré normal à $S m(f)$, diminué de sa section centrale $S m(f)$, peut être rétracté par déformation dans un voisinage de son bord, donc dans un voisinage tel que $J$, dont $K(f)$ est rétracte par déformation. 
Cas des hypersurfaces. Lorsque $p=1, f$ est un plongement, alors il y a lieu de distinguer le cut-locus intérieur $K^{i}$, qui est rétracte par déformation de l'intérieur de la variété plongée (supposée connexe), et le cut-locus extérieur, qui est un rétracte par déformation du complémentaire, lorsqu'on ajoute à $\boldsymbol{R}^{n+1}$ son point à l'infini. (On notera que le cut-locus extérieur peut être vide, comme c'est le cas pour une sphère convexe). Les affirmations précédentes ne sont valables, en principe, que si le cut-locus est générique. Mais, pour un cutlocus quelquonque $K(f)$, il reste vrai que $S m(f)$ est rétracte par déformation du complémentaire de $K(f)$. Il en résulte que l'homologie de $K(f)$ ne dépend que de la classe d'isotopie de $f$; on peut conjecturer qu'il en va de même pour le type d'homotopie faible de $K(f)$.

Cas des sphères plongées. Lorsque $M^{n}$ est une sphère $S^{n}$, on sait, depuis la démonstration par Barry Mazur de la conjecture de Schoênfliess, que l'intérieur de $S^{n}$ est une boule $B^{n+1}$; par suite le cut-locus intérieur est contractile.

Lorsque $n=1$, on en déduit que $K^{i}$ est génériquement un arbre; il a donc au moins deux sommets libres, correspondant à deux minima de rayon de courbure. D'où une démonstration (dûe à $\mathrm{A}$. Weinstein) du théorème des quatre sommets (le rayon de courbure $R$ devant présenter également deux maxima).

Lorsque $n=2$, on en conclut qu'il existe des polyèdres contractiles de dimension deux dont toutes les étoiles sont de type générique. Une adaptation simple d'un théorème dû à $\mathrm{A}$. Weinstein [7] montre qu'il existe des plongements de $S^{2}$ dans $\boldsymbol{R}^{3}$, dont le cut-locus intérieur ne rencontre pas le "conjugate locus", c'est à dire n'a pas de strate libre de type $X^{2}$. Un tel polyèdre ne peut être strictement le classique bonnet croisé (dunce hat), car l'origine n'a pas, dans ce polyèdre, une étoile générique. Mais l'exemple, dû à $E$. R. ReifenbergF.Adams [4] d'une surface minima dans $\boldsymbol{R}^{3}$ qui se rétracte par déformation sur son bord, montre qu'il existe des polyèdres contractiles de dimension deux qui n'ont d'autres singularités que des arêtes triples.

\section{Cut-locus maximal}

On désigne par $M X$ le sous-ensemble fermé des fonctions de $C^{r}(M)$ tel que toute fonction $g$ du complémentaire de $M X$ atteint son maximum en un point critique simple non dégénéré. Si $f$ est une application de $M$ dans $\boldsymbol{R}^{n+p}$, on appelle cut-locus maximal de $f$ la contre-image $K M(f)=\Phi(f)^{-1}(M X)$. Par tout point du complémentaire de $K M(f)$, il passe une normale maximale unique et simple à la partie lisse $\operatorname{Sm}(f)$.

Supposons, pour fixer les idées, que $f$ soit un plongement. Si $z$ n'est pas dans $K M(f)$, soit $q(z)$ le pied de la normale maximale (unique et simple) à l'image $f(M)$ issue de $z$. Comme précédemment, tout point $z^{\prime}$ du prolongement de $q(z) \cdot z$ au-delà de $z$ admet la même normale maximale. De là résulte que le complémentaire de $K M(f)$ se rétracte par déformation à l'infini: dans la situation générique, ce complémentaire est fibré en $S^{p-1} \times \boldsymbol{R}$ sur une partie de $\operatorname{Sm}(f)$, 
chaque fibre étant contenue dans un plan normal à $S m(f)$. Lorsqu'on a affaire à un plongement strictement convexe de $S^{n}$, alors la base de la fibration est $S^{n}$ toute entière, car en tout point de $S^{n}$, il existe une forme linéaire qui atteint son maximum, et une forme linéaire est pratiquement la distance à un point très éloigné. Par suite, en ce dernier cas, le cut-locus maximal est compact, et (génériquement) contractile.

On va établir le

Théorème 2. Pour toute sphère $S^{n}$ plongée dans $\boldsymbol{R}^{n+1}$, le cut-locus intérieur et le cut-locus maximal KM se recontrent.

Observons d'abord qu'il existe une application continue $m$ de $C^{r}\left(S^{n}\right)-W$ dans $S^{n} ; m$ associe à toute fonction $g: S^{n} \rightarrow R$ qui n'est pas dans l'ensemble de Maxwell le point unique où $g$ atteint son minimum absolu. Par ailleurs, l'image $f\left(S^{n}\right)$, si $f$ est un plongement, est disjointe du cut-locus $K(f)$.

Dans le diagramme:

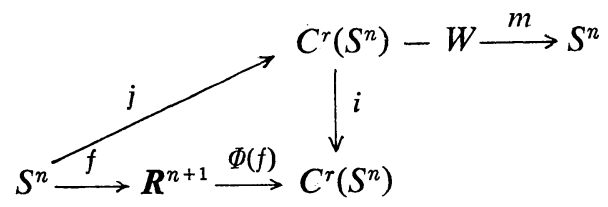

l'application composée $\Phi(f) \circ f$ se relève dans $C\left(S^{n}\right)-W$ et la composée $j=$ $m \circ i^{-1} \circ \Phi(f) \circ f$ est l'identité.

Supposons que le cut-locus intérieur $K^{i}$ soit disjoint du cut-locus maximal $K M$, pour le plongement générique $f$. Alors il existe un voisinage $J$ (défini par exemple par une fonction tapissant $\left.K^{i}\right)$ de $K^{i}$ dans l'intérieur de $f\left(S^{n}\right)$ disjoint de $K M$. Soit $D^{n+1}$ la boule de bord $S^{n}$; on sait qu'il existe un plongement $F$ de $D^{n+1}$ sur l'intérieur de $f\left(S^{n}\right)$ qui prolonge le plongement $f$ au bord. On considère le prodiut $S^{n} \times D^{n+1}$ comme un fibré sur le second facteur $D^{n+1}$, et l'application $m \circ \mathrm{i}^{-1} \circ \Phi(f) \circ f$ comme la section "identique" de ce fibré au-dessus du bord $S_{1}^{n}$ de $D^{n+1}$. Or une telle section s'étend-avec la même définition via $m$-au dessus-du complémentaire de $F^{-1}(J)=J^{\prime}$. Mais sur $J^{\prime}$ on peut définir une autre section de ce fibré, à savoir celle définie par le point $k^{\prime}$ antipodal du maximum $k$ de la fonction distance, point uniquement défini, puisque $J$ est disjoint de $K M$. Mais ôtons du fibré $S^{n} \times D^{n+1}$, restreint à $J^{\prime}$, la section $k$; l'espace restant est un fibré à fibre $S^{n}$ - un point, donc à fibre contractile. Il en résulte que les deux sections, la section $m$ définie sur $\partial J^{\prime}$, et la section $k^{\prime}$ définie sur $K^{i}$, peuvent être reliées par une section continue globale au-dessus de $J^{\prime}$ dans ce même fibré. Mais c'est dire que l'application identique de $S^{n}$ sur elle-même peut être prolongée à $D^{n+1}$, impossibilité bien connue.

Corollaire 2. Pour presque toute courbe fermée simple du plan, il existe un point $x$ qui est centre commun d'un cercle bitangent intérieurement et d'un cercle bitangent extérieurement à la courbe.

Remarque. On éliminera l'hypothèse de généricité dans le résultat du 
théorème 2 en remarquant qu'une application propre entre espaces métriques est une application fermée [3]. On considère la projection canonique $P\left(D^{n+1}, S^{n}\right) \times$ $C^{r}\left(S^{n}\right) \rightarrow C^{r}\left(S^{n}\right)$ : c'est une application propre. Mais dans $D^{n+1} \times C^{r}\left(S^{n}\right)$ la contre-image de $K W$ par $[\Phi(f) \circ F,(\Phi(f))]^{-1}$ est un ensemble fermé; de même pour la contre-image de $M X$; donc aussi pour leur intersection. La projection par $P$ de cet ensemble fermé dans $D^{n+1} \times C^{r}\left(S^{n}\right)$ est fermée dans $C^{r}\left(S^{n}\right)$; mais pour $f$ générique, cette intersection est non-vide; donc l'image par $P$ de l'intersection est dense, dans $C^{r}(M)$, et par suite l'image est $C^{r}(M)$ tout entier. C'est dire que quel que soit $f, K^{i}(f)$ et $K M(f)$ se rencontrent.

\section{Plongements strictement convexes}

Sur le cut-locus intérieur d'une hypersurface, la fonction distance à l'image est définie; pour un tel cut-locus générique, la fonction distance est différentiable dans toute strate. On peut donc parler des points critiques de la fonction distance sur une strate. On peut également parler de minimum (ou maximum) local de cette fonction au voisinage d'un point de $K(f)$.

Proposition 3. Génériquement, les points critiques locaux de la fonction distance sur $K(f)$ ne sont pas sur des strates de conflit, de codimension $>1$, ni sur des strates mixtes.

Ceci vient de ce que le cut-locus est génériquement de dimension $n-1$, et qu'un point critique de la distance est génériquement un point isolé. Supposons dés lors que $f$ soit un plongement strictement convexe de la $n$-sphère $S^{n}$ dans $\boldsymbol{R}^{n+1}$. On a alors la

Proposition 4. Pour le cut-locus d'une sphère strictement convexe, la fonction distance n'a pas de minimum intérieur à une strate de dimension maximum, ni à une strate de conflit ou mixte.

Supposons d'abord la strate plane; si la fonction distance $h$ a un minimum en un point 0 de cet hyperplan, on vérifie immédiatement que l'enveloppe des sphères de centre $x$, de rayon $h(x)$, ne peut être strictement convexe sur la normale en 0 . Il en va de même si la strate est une hypersurface régulièrement plongée en 0: il suffit de le voir par induction sur la dimension, quand la strate est de dimension un; or si la strate est une courbe convexe vers le haut, l'enveloppe des cercles $(x, h(x))$ sera aussi convexe vers le haut sur la normale inférieure en 0 .

Corollaires. Pour une sphère plongée strictement convexe:

i) Le minimum de la distance sur le cut-locus est atteint sur une strate de bifurcation (donc le cut-locus et le conjugate locus se rencontrent).

ii) Si $R$ désigne le minimum d'un rayon de courbure, atteint en un point $x$ de $S^{n}$, la sphère osculatrice en $x$ à $S^{n}$, de rayon $R$, est toute entière dans l'intérieur de $S^{n}$

iii) La fonction distance atteint son maximum en un point unique. 
(Les propriétés ii) et iii) peuvent être démontrées élementairement; elles existent sans doute dans la littérature.)

Désignons par $K_{11}^{m}$ l'ensemble des points $x$ tels que la fonction distance $d(x ; f(y))$ ait la propriété suivante: cette fonction admet au moins deux points critiques d'indice un; les deux qui sont de valeurs la plus basse sont d'égale valeur ou l'un est dégénéré. On va montrer la

Proposition 4. Pour une sphère strictement convexe, le cut-locus $K^{i}$ et l'ensemble $K_{11}^{m}$ se rencontrent.

Si ces ensembles étaient disjoints, alors, en tout point $x$ de $K^{i}$ la fonction distance serait telle que le point d'indice un à valeur la plus basse, s'il existe, serait simple. Mais un tel point existe nécessairement puisqu'en $x \in K^{i}$, la fonction distance a au moins deux minima. Or entre deux minima dont les bassins sont voisins, il y a un col le plus bas qui les met en communication. Puisque $K^{i}$ contient génériquement des strates $X^{2}$, au voisinage de ces strates, on a un col le plus bas qui met ces bassins en communication; pour une métrique riemannienne sur $S^{n}$, on peut trouver des trajectoires de gradients joignant, comme des lignes de thalweg, le col à ces deux minima. Puisque le col et les minima peuvent être prolongés continuement à partir de tout $X^{2}$ du bord, ces lignes de gradients se prolongent également sur tout $K^{i}$ (ceci exclut en fait les strates de conflit de codimension 2 comme les arêtes triples). On peut alors refaire le raisonnement par l'absurde de la démonstration du théorème 2 , en prenant pour section locale le minimum absolu à l'extérieur d'un voisinage $J$ de $K^{i}$, et sur $K^{i}$ le col le plus bas; et on reliera ces sections l'une à l'autre à l'aide des trajectoires de gradients, qui joignent le col aux minima sur $S^{n}$. Ainsi sera définie une section globale conduisant à une extension à $D^{n+1}$ de l'application identique du bord $S^{n}$, d'où contradiction.

Très vraisemblablement, l'étude des propriétés topologiques des cut-loci et des ensembles de bifurcation est d'une extrême richesse et les résultats précédents ne sont guère qu'une première exploration d'un matériel pratiquement inexploré.

L'auteur tient à exprimer sa reconnaissance des fructueuses discussions qu'il à eues avec S. Chern, J. Guckenheimer, S. Łojasiewicz (qui lui a donné des démonstrations élémentaires des propriétés ii) et iii)). Je remercie également A. Weinstein pour une très utile correspondance, et Harry Blum, dont la théorie sur la reconnaissance visuelle des formes a stimulé mon intérêt pour le cutlocus. Le théorème 2, en un cas particulier, a fait l'objet d'un Doctorat de 3ème cycle présenté à l'Université de Paris VII par J. Wandja.

\section{Références}

[1] J. Cerf, La stratification naturelle des espaces de fonctions différentiables réelles et le théorème de pseudo-isotopie, Inst. Hautes Études Sci. Publ. Math. No. 39 (1970) 5-173. 
[2] J. N. Mather, Stable map-germs and algebraic geometry, Lecture Notes in Math. Vol. 197, Springer, Berlin, 1970, 176-193.

[ 3 ] R. Palais, When proper maps are closed, Proc. Amer. Math. Soc. 24 (1970) 835836.

[4] E. R. Reifenberg, Solution of the Plateau problem for m-dimensional surfaces of varying topological type, Acta Math. 104 (1960) 1-92.

[ 5] F. Sergeraert, Thèse, à paraître (Paris-Orsay).

[6] R. Thom, Stabilité structurelle et morphogénèse, Benjamin, New York, à paraître.

[7] A. Weinstein, The cut locus and conjugate locus of a riemannian manifold, Ann. of Math. 87 (1968) 29-41.

Institut des Hautes Etudes Scientifiques 